\title{
Nucleation of Cr precipitates in Fe-Cr alloy under irradiation
}

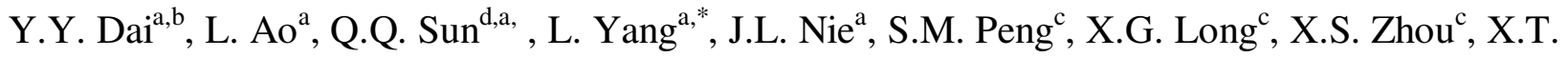

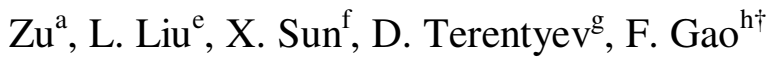 \\ ${ }^{a}$ School of Physical Electronics, University of Electronic Science and Technology of China, Chengdu 610054, China \\ ${ }^{b}$ School of Materials Science and Engineering, Southwest University of Science and Technology, Mianyang 621010, China \\ ${ }^{c}$ Institute of Nuclear Physics and Chemistry, China Academy of Engineering Physics, Mianyang 621900, China \\ ${ }^{d}$ School of Science, Huaihai Institute of Technology, Lianyungang 222005, China \\ ${ }^{e}$ Department of Mechanical, Aerospace, and Nuclear Engineering, Rensselaer Polytechnic Institute, Troy, New York \\ 12180, USA \\ ${ }^{f}$ Pacific Northwest National Laboratory, P.O. Box 999, Richland, WA 99352, USA \\ ${ }^{8}$ SCK-CEN, Nuclear Material Science Institute, Boeretang 200, B-2400, Mol, Belgium \\ ${ }^{h}$ Department of Nuclear Engineering and Radiological Sciences, University of Michigan, Ann Arbor, MI 48109 USA
}

\begin{abstract}
The nucleation of $\mathrm{Cr}$ precipitates induced by overlapping of displacement cascades in $\mathrm{Fe}-\mathrm{Cr}$ alloys has been investigated using the combination of molecular dynamics (MD) and Metropolis Monte Carlo (MMC) simulations. The results reveal that the number of Frenkel pairs increases with the increasing of overlapped cascades. Overlapping cascades could promote the formation of $\mathrm{Cr}$ precipitates in $\mathrm{Fe}-\mathrm{Cr}$ alloys, as analyzed using short range order (SRO) parameters to quantify the degree of ordering and clustering of $\mathrm{Cr}$ atoms. In addition, the simulations using MMC approach show that the presence of small Cr clusters and vacancy clusters formed within cascade overlapped region enhance the nucleation of $\mathrm{Cr}$ precipitates, leading to the formation of large $\mathrm{Cr}$ dilute precipitates.
\end{abstract}

Keywords: Cr precipitate, Fe-Cr alloys, Cascade overlap, Molecular dynamics, Metropolis Mont Carlo

PACS: 02.70.-c, 28.52.Fa

\footnotetext{
* Corresponding author. Tel.: (+86) 028-8320-2130; Fax: (+86) 028-8320-2130; e-mail: yanglildk@uestc.edu.cn

† Corresponding author. Tel: (+1) 509-371-6490; Fax: (+1) 509-371-6242; e-mail: gaofeium@umich.edu
} 


\section{Introduction}

Ferritic/martensitic steels ( 7-12 at.\% Cr) are being considered as candidates for fusion reactor application, because of their inherent radiation damage resistance and of their tensile, impact and creep resistance up to $550{ }^{\circ} \mathrm{C}$ [1]. Recent results have demonstrated that the ferrite separates under neutron irradiation into two isomorphous phases, Fe-rich $(\alpha)$ and $\mathrm{Cr}$-rich $\left(\alpha^{\prime}\right)$ phases, when the $\mathrm{Cr}$ content of the ferritic matrix is larger than a critical threshold value of $\sim 7.2$ at.\% at $325{ }^{\circ} \mathrm{C}$ [2]. The presence of Cr-rich precipitates has been shown to contribute significantly to the irradiation-induced hardening [2]. Therefore, the understanding of the nucleation, growth and coarsening of $\mathrm{Cr}$ precipitates under irradiation is of importance for safe exploitation of F/M steels under irradiation.

In the last decade much of the work has been carried out related to displacement cascades, and has focused on the defects in the matrix of Fe-Cr alloys [3-8]. In addition, the effect of the overlapping of single $5 \mathrm{keV}$ displacement cascades on defect production and ordering in $\mathrm{Fe}-\mathrm{Cr}$ alloys was studied by molecular dynamics (MD) simulation [9]. However, the nucleation and growth of Cr precipitates were not observed due to MD time span limiting the defect diffusion. It is of great interest to explore if the defects created by displacement cascades can enhance the nucleation of $\mathrm{Cr}$ precipitates in $\mathrm{Fe}-\mathrm{Cr}$ alloys.

Experimentally, it was observed that irradiation can accelerate the phase separation, and thus enhances the formation of chromium-rich precipitates, as revealed by magnetic domain characteristics [10]. There are many factors that will influence the microstructural evolution of $\mathrm{Fe}-\mathrm{Cr}$ alloys under irradiation. The content of $\mathrm{Cr}$ in $\mathrm{Fe}-\mathrm{Cr}$ alloys can be controlled to improve their resistance against corrosion and thermal creep, but also to influence the extent of radiationinduced strengthening and embrittlement $[11,12]$. The increase in irradiation temperature results 
in high defect mobility and enhanced recombination, which causes heightened rates of recombination or annihilation at sinks and accelerated growth/shrinkage of clusters [13]. The supersaturation of point defects is one of the factors for the acceleration of $\mathrm{Cr}$ precipitates, but the overlapping of cascades that affects the formation and distribution of defect clusters may be another important factor to control the nucleation of $\mathrm{Cr}$ precipitates, thus affecting the kinetics of short range order (SRO) parameter and decomposition. In this paper we focus on the effect of the cascade overlapping on the $\mathrm{Cr}$ precipitates.

The nucleation and growth of $\mathrm{Cr}$ precipitates are clearly multiscale phenomena, and no single method could model the whole range of time and length scales. Thus, the combination of simulation techniques should provide a possible pathway to explore this issue, particularly under extreme conditions. The molecular dynamics simulation stands for a computationally intensive method for modeling atomic systems on the time and length scales appropriate to displacement cascades. As another widespread numerical technique used to study the thermodynamically favored systems of $N$ atoms, Metropolis Monte Carlo (MMC) technique allows the final equilibrium to be achieved within an affordable computing time. In order to understand the nucleation of $\mathrm{Cr}$ precipitates under irradiation in $\mathrm{Fe}-\mathrm{Cr}$ alloys, atomistic simulations using $\mathrm{MD}$ and MMC methods were performed. The present paper explores the nucleation of $\mathrm{Cr}$ precipitates under overlapping displacement cascades. In reality, the irradiation dose is relatively low, particularly associated with neutron irradiation, but there may still be an overlap effect. In Sec. II, the methodology for the present study, including interatomic potential, molecular dynamics, Metropolis Monte Carlo and analysis method, is presented, while the defect production due to cascade overlap is presented in Sec. III, examining defect configurations, defect clustering and possible aggregation of $\mathrm{Cr}$ atoms by analyzing the short range ordering (SRO) parameters as a 
function of cascade overlap events. The changes of $\mathrm{Cr}$ ordering as a function of MMC steps, as well as the atomic configurations of $\mathrm{Cr}$ precipitates and vacancies before and after MMC simulation, will be explored and the consequences of these findings will be discussed.

\section{Methodology}

In terms of accelerated dynamics process, there have been a large number of techniques developed, such as metadynamics [14] and temperature accelerated dynamics [15]. Also, kinetic Monte Carlo (KMC) method has been used to extend the time scale of molecular dynamics simulation and has been applied to study the defect accumulation in irradiated materials [16] and defect recovery [17]. A different kind Monte Carlo method is Metropolis Mont Carlo (MMC) that leads to a system in equilibrium, sampling configuration space, rather than following the physical path toward this equilibrium. However, it has been demonstrated that MMC is suited to drive the system towards thermodynamic equilibrium [18]. In the present study, we have combined $\mathrm{MD}$ and $\mathrm{MMC}$ to investigate the nucleation of $\mathrm{Cr}$ precipitates in $\mathrm{Fe}-\mathrm{Cr}$ alloys under overlapping displacement cascades. MD method is applied to study cascade overlap, whereas MMC approach is employed to get the thermodynamic equilibrium configuration after overlapping 10 cascades and study how defects affect the precipitates of Cr. The details of each method will be described in the following sections. Ideally, MMC approach should be applied after each cascade overlap to achieve the thermodynamic equilibrium configuration, and then, continue with another cascade overlap. Several cascade overlaps and MCC simulations are alternatively carried, but we do not observe the formation of a $\mathrm{Cr}$ precipitate within the core of the cascade, even though a few Cr-rich regions far away from the cascades are thermodynamically formed. This may suggest that the high defect concentration or high vacancy 
concentration within the cascade core needs to be established for the formation of $\mathrm{Cr}$ precipitates. This approach slightly changes the number of defects produced and clustering, but does not affect the enhanced nucleation of $\mathrm{Cr}$ precipitates induced by cascade overlap overall.

The interatomic potential for Fe-Fe interaction was taken from the work of Ackland and Mendelev [19], which provides the reasonable description of point defects and is used to simulate cascade damage in $\mathrm{Fe}$ [20]. The interactions for $\mathrm{Cr}$ and $\mathrm{Fe}-\mathrm{Cr}$ are described using a twoband second moment model derived by Olsson et al [21]. These potentials have been employed to simulate displacement cascades in $\mathrm{Fe}-\mathrm{Cr}$ systems [6], and proven to provide a reasonably good description of the interaction between $\mathrm{Cr}$ atoms and point defects in a ferritic matrix, as well as the migration of Cr-vacancy clusters and interstitial $\mathrm{Cr}$ in $\alpha$-Fe [22]. Thus, these potentials should be reliable in the study of cascade overlap, defect production and MMC simulations of defect enhanced nucleation of $\mathrm{Cr}$ precipitates in $\mathrm{Fe}-\mathrm{Cr}$ alloys.

\subsection{Molecular dynamics simulation}

MD simulations were performed using the modified version of MOLDY code [23]. The simulation cell consisted of $50 \mathrm{a}_{0} \times 50 \mathrm{a}_{0} \times 50 \mathrm{a}_{0}\left(\mathrm{a}_{0}=2.8553 \AA\right)$ bcc unit cells $(250,000$ atoms $)$, randomly distributed 10 at.\% and 15 at.\% $\mathrm{Cr}$ in Fe, respectively, as shown in Table 1, where $\mathrm{Fe}$ atoms are randomly replaced by $\mathrm{Cr}$ atoms, forming substitution $\mathrm{Cr}$ atoms in bcc $\mathrm{Fe}$. Two temperatures are considered in the present work, i.e. $300 \mathrm{~K}$ and $600 \mathrm{~K}$, and periodic boundary conditions are imposed along three main directions, with a time step of $1 \mathrm{fs}$. According to the solubility results of Bonny et al. [24], the Cr concentration of 10 at.\% at $300 \mathrm{~K}$ and $600 \mathrm{~K}$ is not supersaturated, and the 15 at.\% $\mathrm{Cr}$ at $600 \mathrm{~K}$ is very close to the $\mathrm{Cr}$ solubility limit. To simulate a cascade, the block was first quenched to $0 \mathrm{~K}$ to obtain the minimum energy configuration, then 
rescaled to the required temperature and equilibrated for at least $10 \mathrm{ps,} \mathrm{establishing} \mathrm{the}$ equilibrium phonon modes before a primary knock-on atom (PKA) event is launched.

A cascade was started by imparting a given kinetic energy $E_{p}(10 \mathrm{keV})$ to a PKA at a distance of $3 \mathrm{~nm}$ from the center of the MD block. The purpose of the current study is to understand how defects and defect clusters affect the nucleation of $\mathrm{Cr}$ precipitates, so that a high-index direction $<351>$, which is located in the middle of a standard stereographic unit triangle, is selected for initiating a PKA. This direction will avoid channeling, forming possible compact cascade geometry, which might lead to the most dominant effect on cascade overlap. Interstitials and vacancies were identified and counted using displacement analysis. If an atom is displaced from its lattice site by at least $30 \%$ of the lattice constant, this atom is considered as an interstitial; if there is no atom within $30 \%$ of the lattice constant of a lattice site, the site is assumed to be a vacancy. After the PKA was initiated, the crystal was allowed to evolve for 20 ps, the crystal containing the defects created by this cascade was quenched to $0 \mathrm{~K}$, following which it was reequilibrated at the required temperature for $20 \mathrm{ps}$ and then, second cascade (the overlap event) was initiated. This approach will be applied to the rest of the cascade overlap with ten overlapped events. The multiple PKA positions were repeatedly initiated at random locations for cascade events, but at a same radius of $3 \mathrm{~nm}$ from the center of the MD block and toward the center with a similar direction of $<351>$. Because the damaged regions created by the previous PKAs are close to the center of the MD block, the simulation procedure described here allows the maximum effect of the thermal spike on those damaged regions

\subsection{Metropolis Monte Carlo simulation}

The molecular dynamics simulations of the tenth cascade overlap are followed by MMC simulations. The MMC simulations are performed within the constant number of atoms, volume 
and temperature (NVT) ensemble, and the required temperature remains the same as in the MD simulations. Two types of trials are considered: (i) random displacement of all atoms from their current positions, by which the lattice relaxation and vibration entropy are taken into account; (ii) randomly swapping of lattice sites between atoms of different kinds. The detailed information about the two MMC trials is described in Ref. 25. Maximum allowed displacement of an atom is $0.015 \AA$ along $\mathrm{x}, \mathrm{y}$ and $\mathrm{z}$ directions. The new configuration is accepted if it leads to energy decrease or with a probability evaluated from the relevant Boltzmann distribution if the system energy increases. The final configurations reported here correspond to the averaged atomic positions over the last $2 \times 10^{5} \mathrm{MMC}$ steps.

\subsection{Analysis}

We have used the so called cluster connectivity analysis [26] to identify defects and defect clusters, where the second-nearest-neighbor distance was used for vacancies, but the distance extended to the third-nearest-neighbor was used for analyzing self-interstitial atoms. If a cluster consists of 15 self-interstitial atoms (SIAs) and 10 empty lattices, it was counted as a 5-SIAs cluster. The conservation of defects created is checked to make sure that the number of vacancies is equal to the number of SIAs, which accounts for the number of Frenkel pairs generated. The changes of $\mathrm{Cr}$ ordering are analyzed using SRO parameters defined by Cowley [27]. The $k$ th shell SRO parameter and the coordination number-weighted average of the SRO parameter are defined as those in Ref. 28. The $k$ th shell SRO parameter of a $\mathrm{Cr}$ atom is defined as:

$$
\alpha_{C r}^{(k)}=1-\frac{z_{F e}^{k}}{z_{t o t}^{k}\left(1-C_{C r}\right)},
$$

where $z_{F e}^{k}$ denotes the number of Fe atoms in the $k$ th shell, $z_{\text {tot }}^{k}$ is the total number of atoms in the same shell, and $\boldsymbol{C}_{\boldsymbol{C}}$ is the overall $\mathrm{Cr}$ concentration. The coordination number-weighted average of the parameter $\mathrm{SRO}_{12}$ is defined as:

$$
\beta=\frac{8 \alpha_{C r}^{(1)}+6 \alpha_{C r}^{(2)}}{14}
$$




\section{Results and Discussion}

\subsection{Cascade overlap}

\subsubsection{Defect production}

As described above, the crystal containing the defects created by each cascade was quenched to $0 \mathrm{~K}$ and then rescaled to the required temperature for continuing cascade overlap. Figs. 1(a), (b) and (c) show the defect distributions after the first cascade, seventh and tenth overlapped cascades at $300 \mathrm{~K}$, with 10 at.\% Cr concentration, respectively. The green and blue spheres represent $\mathrm{Fe}$ and $\mathrm{Cr}$ interstitials, respectively, and the red represent vacancies. After the first cascade, the most SIAs are single interstitials, while a small cluster of vacancies (containing 11 vacancies) is formed at the center of the cascade. In this work, the number of the vacancies in a vacancy cluster is accounted for by the total number of the vacancies minus that of $\mathrm{Fe}$ and $\mathrm{Cr}$ interstitials in a large cluster formed at the center of the damaged region. After the seventh overlapped cascade, several small interstitial and vacancy clusters are formed, but large vacancy clusters are not observed. However, it is evidenced that the size of interstitial clusters increases with increasing the overlapped cascades, but only a few $\mathrm{Cr}$ interstitials are produced, even after ten overlapped cascades. The similar phenomena are observed for the $\mathrm{Fe}-\mathrm{Cr}$ system with $15 \% \mathrm{Cr}$ concentration. After the tenth overlapped cascade, it is clearly seen that a large vacancy cluster consisting of 61 vacancies is formed at the center of the damage region, with several large interstitial clusters produced at the periphery of the damage region. It is known that the migration energy of a vacancy in $\alpha$-Fe with the present potential is about $0.62 \mathrm{eV}$ [19], which suggests that the vacancies are immobile at $300 \mathrm{~K}$ within typical MD time span. Besides, the formation of vacancy clusters is slightly affected by the presence of $\mathrm{Cr}$ [29]. Therefore, the formation of the 
large vacancy cluster may be due to the irradiation-enhanced migration of vacancies, which is associated with the high temperature formed at the cores of cascades, sometimes higher than the melting temperature [30]. It is also observed that a large vacancy cluster at $600 \mathrm{~K}$ is formed much earlier than that at $300 \mathrm{~K}$, which supports the mechanism proposed here.

The number of Frenkel pairs versus the number of the overlapped cascades is shown in Fig. 2. The Frenkel pairs at the end of ten overlapped cascades at $600 \mathrm{~K}$ are 74 and 73 for Fe-10 at. $\%$ $\mathrm{Cr}$ and $\mathrm{Fe}-15$ at.\%, respectively. In order to investigate the effects of different irradiation temperatures on defect production, we have simulated cascade overlap at 300 and $600 \mathrm{~K}$ for the same $\mathrm{Cr}$ concentration. It can be clearly seen that the effect of the irradiation temperature on the number of Frenkel pairs is small, which is similar to that found in pure Fe, as the temperature increases from 100 to $600 \mathrm{~K}$ [31]. Generally, the number of Frenkel pairs increases with the number of the overlapped cascades.

Among those vacancies produced in the ten overlapped cascades, a few are still single vacancies, but most form vacancy clusters. The number of vacancy clusters and the corresponding number of vacancies in each vacancy cluster defined as on the number of the overlapped cascades is presented in Fig. 3, which clearly demonstrates that the size of the largest vacancy cluster increases after the eighth cascade overlap. For example, the size of the largest vacancy cluster increases from 25 to 61 after the eighth and tenth overlapped cascade in the Fe10 at.\% Cr alloy at $300 \mathrm{~K}$, as shown in Fig. 3(a). However, it is observed that the vacancy clusters may be dissolved due to the impact of cascade overlap. For example, there is only a vacancy cluster containing 30 vacancies in the $\mathrm{Fe}-15$ at.\% $\mathrm{Cr}$ alloy at $600 \mathrm{~K}$ after the fourth overlap cascade, and there are three vacancy clusters containing 2,3 and 6 vacancies after the sixth overlap cascade, as shown in Fig. 3(c). Also, it is of interest to note that large vacancy 
clusters are formed after ten overlapped cascades with different $\mathrm{Cr}$ concentrations at the different temperatures considered in this work.

\subsubsection{Degree of Cr ordering}

The ordering in the $\mathrm{Cr}$ distribution can be described by SRO parameters. The $k$ th shell SRO parameters are defined by Cowley to express the interaction of a given atom in an alloy with the $k$ th shell of atoms surrounding it. The detailed definition is given in ref. [28]. The dependence of the first and second shell SRO parameters on cascade overlap is shown in Figs. 4(a) and (b) respectively, where the unit of all the SRO parameters is scaled to 0.01. As can be seen in Fig. 4(a), the first shell SRO parameter increases with increasing the overlapped cascades and becomes larger at $600 \mathrm{~K}$ than at $300 \mathrm{~K}$ after $5^{\text {th }}$ overlapped cascade in $\mathrm{Fe}-10$ at.\%, which suggests that increasing temperature enhances the nucleation of $\alpha^{\prime}$ phase ( $\mathrm{Cr}$ rich phase). Also, the higher $\mathrm{Cr}$ concentration leads to fast increase in the first shell SRO parameters with increasing overlapped cascades. Especially, after the $6^{\text {th }}$ overlapped cascade, it becomes positive in $\mathrm{Fe}-15$ at.\% $\mathrm{Cr}$ alloy, which indicates the segregation of $\mathrm{Cr}$ atoms. Furthermore, the present results demonstrate that the cascade overlap promotes the clustering of $\mathrm{Cr}$ in the first shell of $\mathrm{Fe}$ $\mathrm{Cr}$ alloys. It is concluded that the higher temperature and $\mathrm{Cr}$ concentration would advance the segregation of $\mathrm{Cr}$ atoms in the first shell of $\mathrm{Fe}-\mathrm{Cr}$ alloys during overlapping cascades.

For the second shell, the SRO parameter shows small fluctuation during overlapping cascades, and remains essentially the same on average as illustrated in Fig. 4(b). Even with the cascade overlap, the second shell SRO parameter is almost negative, which means that the long range ordering is weak. These results indicate that the alloys mainly consist of the $\alpha$ phases (Ferich phases) and $\mathrm{Cr}$ atoms tend to be ordered in the first shell. In $\mathrm{Fe}-15$ at.\% $\mathrm{Cr}$ alloy, the second SRO parameter approaches zero, but becomes positive during the $7^{\text {th }}$ and $8^{\text {th }}$ overlapped 
cascades. The high $\mathrm{Cr}$ concentration may promote the clustering of $\mathrm{Cr}$ atoms in the second shell. In addition, the second shell $\mathrm{SRO}$ parameter at $300 \mathrm{~K}$ is smaller than at $600 \mathrm{~K}$ in $\mathrm{Fe}-10$ at. $\% \mathrm{Cr}$ alloy, which suggests that the higher temperature could enhance the clustering of $\mathrm{Cr}$ atoms in the second shell. It should be noted that the second shell SRO parameter is independent of the cascade overlap.

The short-range order of a system can be also described by the $\mathrm{SRO}_{12}$ parameter defined as the coordination number-weighted average of the SRO parameters for the first and second neighbor shells. The dependence of the $\mathrm{SRO}_{12}$ on cascade overlap is shown in Fig. 4(c), which shows that the $\mathrm{SRO}_{12}$ increases with increasing the overlapped cascades. In Fe-10 at.\% $\mathrm{Cr}$ alloy, $\mathrm{SRO}_{12}$ is negative, denoting the presence of short range ordering of $\mathrm{Cr}$ atoms. In $\mathrm{Fe}-15$ at.\% $\mathrm{Cr}$ alloy, $\mathrm{SRO}_{12}$ becomes positive after the seventh overlapped cascade, which indicates that $\mathrm{Cr}$ atoms start forming clusters. Moreover, the $\mathrm{SRO}_{12}$ at $600 \mathrm{~K}$ is larger than at $300 \mathrm{~K}$ in $\mathrm{Fe}-10$ at. $\%$ $\mathrm{Cr}$ alloy, but is smaller than in $\mathrm{Fe}-15$ at.\% Cr alloy. These results, along with the analysis above, demonstrate that the cascade overlap could promote the clustering of $\mathrm{Cr}$ atoms in $\mathrm{Fe}-\mathrm{Cr}$ alloys. In addition to the effects of cascade overlap, SRO parameters are dependent on both temperature and concentration of $\mathrm{Cr}$ atoms, which is consistent with the results obtained in Ref. 28.

It is well known that the dose rate is an important parameter controlling the defect recombination and interaction within cascades. However, the development of a method to simulate the experimental dose rate is extremely challenging. Although the MD and MMC have been combined to study in-cascade nucleation of $\mathrm{Cr}$ precipitates in $\mathrm{Fe}-\mathrm{Cr}$ Alloys, the dose rate effect cannot be addressed. However, the MMC method can be used to predict the thermodynamic equilibrium configuration that a system could achieve for the steps simulated, which may underestimate annealing of the cascade for a given dose rate. However, it is expected that 
the dose rate may affect the results slightly, but does not alter the major conclusions in the present study.

\subsection{Simulation}

\subsubsection{Cr ordering}

As can be seen in Fig. 1 and Fig. 2, the number of vacancies produced in the first several cascades is small, and only a few small vacancy clusters are formed. It is well known that the binding energy of a $\mathrm{Cr}$ atom with a vacancy or a small vacancy cluster is very small [22], and thus, it is unlikely that these vacancies would enhance the segregation of $\mathrm{Cr}$ atom, even with much longer simulation time than allowed in the MD runs. However, it is clearly shown that a large vacancy cluster, containing 61, 51 and 39 vacancies in the $\mathrm{Fe}-10$ at.\% $\mathrm{Cr}$ at $300 \mathrm{~K}$ and 600 $\mathrm{K}$, and $\mathrm{Fe}-15$ at. $\% \mathrm{Cr}$ at $600 \mathrm{~K}$ respectively, is formed after the tenth overlapped cascade, but the segregation of $\mathrm{Cr}$ atoms to this cluster does not occur, which may be due to the short time scale of MD simulations. Here, MMC method has been employed to achieve the equilibrium configuration of the system, thus understanding the effects of irradiation on the nucleation of $\mathrm{Cr}$ precipitate. The thermodynamically equilibrium configurations of $\mathrm{Fe}-\mathrm{Cr}$ alloys are simulated using MMC approach, with $2 \times 10^{5}$ MMC steps. The evolution of the first shell SRO parameter, the second shell SRO parameter and the $\mathrm{SRO}_{12}$ as a function of the MMC steps is shown in Figs. 5(a) - (c), respectively. All the SRO parameters increase with the increase of MMC steps. Initially, the SRO parameters show a fast increases, but become slow after $5 \times 10^{4} \mathrm{MMC}$ steps. The SRO parameters become positive after a few hundred MMC steps, correlating to the formation of Cr clusters. In Fe-15 at.\% Cr system, the SRO parameters are higher than in Fe-10 at. $\% \mathrm{Cr}$, and the increasing rate of the SRO parameters is faster in $\mathrm{Fe}-15$ at.\% $\mathrm{Cr}$, compared with $\mathrm{Fe}-10$ at.\% Cr. In the Fe-10 at.\% Cr system, the SRO parameters are slightly higher at $300 \mathrm{~K}$ than those at $600 \mathrm{~K}$, and the increasing rate of the SRO parameters is also slightly faster at 300 
$\mathrm{K}$. These results indicate that the decrease of temperature somewhat increases the probability of $\mathrm{Cr}$ clustering, because of the low binding energies of $\mathrm{Cr}$ atoms to a cluster [21,22], leading to $\mathrm{Cr}$ atoms dissociation from the cluster at high temperatures.

According to Figs. 5 (a) - (c), it is clear that the SRO parameters are both dependent on temperature and $\mathrm{Cr}$ concentration, which is consistent with Ref. 28. With the increase of $\mathrm{Cr}$ concentration, the SRO parameters increase, which may suggest that the increase in $\mathrm{Cr}$ concentration promotes the formation of Cr clusters. However, the effect of the concentration of $\mathrm{Cr}$ atoms on the SRO parameters is somewhat larger as compared to the effect of temperature. After 10 overlapped cascades, the thermodynamically favorable configuration of $\mathrm{Fe}-\mathrm{Cr}$ alloys contains the Cr clusters.

\subsubsection{Cr Precipitates under irradiation}

As an illustration, the distribution of $\mathrm{Cr}$ atoms near the largest vacancy cluster before and after the MMC simulation is presented in Figs. 6 (a) - (f). The large vacancy cluster is formed at the center of displacement cascades after 10 overlapped cascades, but only a $\mathrm{Cr}$ precipitate with $24 \mathrm{Cr}$ atoms is formed. However, the several $\mathrm{Cr}$ precipitates are homogenously distributed over the whole matrix in $\mathrm{Fe}-15$ at.\% $\mathrm{Cr}$, and the largest size of $\mathrm{Cr}$ precipitates contains $65 \mathrm{Cr}$ atoms. The $\mathrm{Cr}$ precipitates mentioned in the present work are the $\mathrm{Cr}$-rich precipitates, which consist of $\mathrm{Cr}$ and $\mathrm{Fe}$ atoms. Therefore, the concentration of $\mathrm{Cr}$ atoms dramatically affects the formation of Cr precipitates in the overlapped cascades. After $2 \times 10^{5} \mathrm{MMC}$ steps, more $\mathrm{Cr}$ precipitates were formed in Fe-Cr alloys, which is consistent with the analysis of SRO parameters above.

Figs. 6(b) and (d) demonstrate the configurations of $\mathrm{Cr}$ atoms and vacancies in $\mathrm{Fe}-10$ at.\% $\mathrm{Cr}$

after $2 \times 10^{5}$ MMC steps, at 300 and $600 \mathrm{~K}$, respectively. More $\mathrm{Cr}$ atoms migrate towards the vacancy-rich region at $600 \mathrm{~K}$ compared with that at $300 \mathrm{~K}$ leading to the formation of a larger $\mathrm{Cr}$ 
precipitate at the center of the damaged region, as compared with that at $300 \mathrm{~K}$. This may be due to the high concentration of vacancies at the cascade core induced by the cascades and thus, the $\mathrm{Cr}$ atoms could migrate through a vacancy diffusion mechanism, which is consistent with the result found by Chen et al [22]. It is clearly shown that there are large Cr precipitates at the core of the damage region, and these precipitates are diluted by vacancies. It is generally accepted that the cluster binding energy increases as the number of vacancies in the cluster increases but it decreases as the number of $\mathrm{Cr}$ atoms increases [22]. This suggests that adding vacancies to the small cluster can stabilize the $\mathrm{Cr}$ atoms in the cluster and that adding $\mathrm{Cr}$ atoms in the cluster leads to the screening of $\mathrm{Cr}$ atoms from each other. Also, it should be noted that Cr-rich precipitates ( $\alpha^{\prime}$ phases) are homogenously distributed in the matrix. These results indicate that the main factor controlling the formation of $\mathrm{Cr}$ precipitates is not only associated with the presence of vacancies, but also correlated to the small $\mathrm{Cr}$ clusters formed during overlapping cascades.

As the $\mathrm{Cr}$ concentration increases from 10 to 15 at.\%, the number of $\mathrm{Cr}$ precipitates dramatically increase from 40 to 390, as shown in Figs. 6(f). In the Fe-15 at.\% Cr system, the size of largest $\mathrm{Cr}$ precipitates consists of $172 \mathrm{Cr}$ atoms after $2 \times 10^{5} \mathrm{MMC}$ steps, as compared with $33 \mathrm{Cr}$ atoms of the largest precipitate in the $\mathrm{Fe}-10$ at.\% $\mathrm{Cr}$. The high concentration of $\mathrm{Cr}$ atoms enhances the formation and growth of $\mathrm{Cr}$ precipitates during overlapping cascades, as demonstrated by the larger SRO parameters of $\mathrm{Fe}-15 \% \mathrm{Cr}$. The $\mathrm{Cr}$ concentration dramatically affects the nucleation of $\mathrm{Cr}$ precipitates during overlapping cascades, and the number and size of Cr precipitates increase with increasing $\mathrm{Cr}$ concentration.

It should be noted that there is an important effect of cascade overlap on the damaged region, enhancing the formation of vacancy clusters. As shown in Fig. 6, these vacancy clusters provide the initial sites for nucleating $\mathrm{Cr}$ precipitates, where the nearby $\mathrm{Cr}$ atoms preferentially migrate 
into the vacancy clusters during MMC simulation. It should be emphasized that the formation of the $\mathrm{Cr}$ precipitates at the centers of the damaged regions is observed at all the $\mathrm{Cr}$ concentrations and all the temperatures considered, which may further account for the decrease of SRO parameters described above. However, some small Cr-rich clusters are formed, but they are observed only at higher Cr concentration and higher temperature (Figs. 6(e) and (f)). Moreover, these small clusters are already nucleated during the cascade overlap (Fig. 6(e)), rather than that during aging by MMC. Also, the size of the $\mathrm{Cr}$ precipitates formed at the vacancy clusters is much larger than the small $\mathrm{Cr}$-rich clusters. These results well demonstrate the nucleation of $\mathrm{Cr}$ precipitates enhanced by cascade overlap.

\section{Conclusion}

The MD and MMC methods have been combined to study $\mathrm{Cr}$ precipitates during overlapping cascades in $\mathrm{Fe}-\mathrm{Cr}$ alloys, with different concentrations of $\mathrm{Cr}$ atoms and at different temperatures. The number of Frenkel pairs increases with the increasing of the overlapped cascades. The number of vacancies and the size of vacancy clusters increase with increasing the overlapped cascades. The SRO parameters are used to analyze the formation of $\mathrm{Cr}$ precipitates during overlapping cascades. With increasing the number of the overlapped cascades, the SRO parameters increase, which indicates that the cascade overlap could promote the formation of $\mathrm{Cr}$ precipitates in $\mathrm{Fe}-\mathrm{Cr}$ alloys.

The thermodynamically favorable configurations of Fe-Cr alloys after 10 overlapped cascades are simulated using MMC technique. The vacancy clusters formed within cascade overlapped damage enhance the nucleation of $\mathrm{Cr}$ precipitates, leading to the formation of large $\mathrm{Cr}$ dilute precipitates. Also, the small $\mathrm{Cr}$ clusters formed during overlapping cascades act as the nucleus to 
form large Cr-rich precipitates ( $\alpha^{\prime}$ phases), particularly at high $\mathrm{Cr}$ concentrations. The number and size of these $\mathrm{Cr}$ precipitates increase with increasing the $\mathrm{Cr}$ concentration and are homogenously distributed in the matrix of $\mathrm{Fe}-\mathrm{Cr}$ alloys.

\section{Acknowledgments}

Y.Y. Dai is grateful for the support by the program for specialized research fund for the doctoral program (14zx7152). L. Ao, Q.Q. Sun, L. Yang and X.T. Zu are grateful for the support by the National Natural Science Foundation of China — NSAF (Grant No: 10976007, U1430109). J.L. Nie is grateful for the support by the National Natural Science Foundation of China (Project No. 51201026). S.M. Peng, X.G. Long and X.S. Zhou are grateful for the Science and Technology Foundation of China Academy of Engineering Physics (Grant No: 2010A0301011). F. Gao, L. Liu and X. Sun are grateful for the support by the US Department of Energy, Office of Nuclear Energy (Nuclear Energy University Program), under Contract DE-AC06-76RLO 1830. . 


\section{Reference}

[1] R.L. Klueh, D.R. Harries, ASTM Monograph 3, American Society for Testing and Materials, West Conshohocken, PA, 2001.

[2] M.H. Maton, Y. de Carlan, G. Geoffroy, X. Averty, A. Alamo, C.H. de Novion, J. Nucl. Mater. 312 (2003) 236-248.

[3] A. Caro, D.A. Crowson, M. Caro, Phys. Rev. Lett. 95 (2005) 075702.

[4] D.A. Terentyev, L. Malerba, R. Chakarova, K. Nordlund, P. Olsson, M. Rieth, J. Wallenius, J. Nucl. Mater. 349 (2006) 119-132.

[5] K. Vortler, C. Bjorkas, D. Terentyev, L. Malerba, K. Nordlund, J. Nucl. Mater. 382 (2008) 24-30.

[6] C. Bjorkas, K. Nordlund, L. Malerba, D. Terentyev, P. Olsson, J. Nucl. Mater. 372 (2008) $312-317$.

[7] Jae-Hyeok Shim, Hyon-Jee Lee, Braian D. Wirth, J. Nucl. Mater. 351 (2006) 56-64.

[8] L. Malerba, D. Terentyev, P. Olsson, R. Chakarova, J. Wallenius, J. Nucl. Mater. 329-222 (2004) 1156-1160.

[9] K. Vortler, N. Juslin, G. Bonny, L. Malerba, K. Nordlund, J. Phys.: Condens. Matter 23 (2011) 355007.

[10] Yasuhiro Kamada, Hideo Watanabe, Seiji Mitani, Jitendra Narayana Mohapatra, Hiroaki KiKuchi, Satoru Kabayashi, Masaki Mizuguchi, Koki Takanashi, J. Nucl. Mater. 442 (2013) S861-S864.

[11] K. H. Mayer and F. Masuyama. "The development of creep-resistant steels." Creep-resistant steels, ed. F. Abe, T.-K. Kern, R. Viswanathan, Woodhead Publ. Ltd, Cambridge, England (2008): 15-71. 
[12] A.Kohyama, A.Hishinuma, D.S.Gelles, R.L.Klueh, W.Dietz, K.Ehrlich, J.Nucl.Mater. 138 (1996) 233-237.

[13] Christopher D. Hardie, Ceri A. Williams, S. Xu, Steve G. Roberts, J.Nucl.Mater. 439 (2013) $33-40$.

[14] A. Laio and M. Parrinello, Proc Natl Acad Sci USA, 99 (2002) 12562.

[15] M. Sorensen and A Voter. J. Hcem Phys 112 (2000) 9599.

[16] E. Alonsoa, M.-J. Caturlaa, T. Diaz de la Rubiaa, J.M. Perladob, J. Nucl. Mater. $276(2000) 221$.

[17] Z.W. Rong, F. Gao, William J. Weber and Gerhard Hobler, J. Appl. Phys. 102 (2007) 103503.

[18] L. Malerba, A. Caro and J. Wallenius, J. Nucl. Mater. 328 (2008) 112-125.

[19] G. Ackland, M. Mendelev, D. Srolovitz, S. Han, and V. Barashev, J. Phys.: Condens. Matter 16 (2004) S2629.

[20] A. F. Calder, D. J. Bacon, A. V. Barashev, and Yu. N. Osetsky, J.Nucl. Mater. 382 (2008) 91.

[21] P. Olsson, J. Wallenius, C. Domain, K. Nordlund and L. Malerba, Phys. Rev. B 72 (2005) 214119.

[22] D. Chen, F. Gao, W. Y. Hu, S. Y. Hu, D. Terentyev, X. Sun, H. L. Heinisch, C. H. Henager, and M. A. Khaleel, Phys. Rev. B, 81 (2010) 064101.

[23] F. Gao, D. J. Bacon, P. E. J. Flewitt, T. A. Lewis, J. Nucl. Mater. 249 (1997) 77.

[24] G. Bonny, R.C. Pasianot, D. Terentyev, L. Malerba, Philos. Mag., 91(2011) 1724-1746.

[25] Maxie Eckert, Erik Neyts and Annemie Bogaerts, Cryst. Eng. Comm. 11 (2009) 1597-1608.

[26] Erez Hartuv, Ron Shamir, Inform. Process. Lett. 76 (2000) 175-181. 
[27] J.M. Cowley, Phys. Rev. 77 (1950) 669-675.

[28] Paul Erhart, Alfredo Caro, Magdalena Serrano de Caro and Babak Sadigh, Phys. Rev. B, 77 (2008) 134206.

[29] C. Bjorkas, K. Nordlund, L. Malerba, D. Terentyev, P. Olsson, J. Nucl. Mater. 372 (2008) $312-317$

[30] T. Diaz de la Rubia, R. S. Averback and H. Hsieh, J. Mater Res. 4(1989)579.

[31] D.J. Bacon, A.F. Calder, F. Gao, J. Nucl. Mater. 251 (1997) 1-12. 
Table 1. Recoil energy, number of the overlapped cascades, box size, and number of atoms used in randomly distributed $\mathrm{Fe}-10$ at.\% $\mathrm{Cr}$ and $\mathrm{Fe}-15$ at.\% Cr systems.

\begin{tabular}{cccccc}
\hline & $\begin{array}{c}\text { Energy } \\
(\mathrm{keV})\end{array}$ & $\begin{array}{c}\text { Temperature } \\
(\mathrm{K})\end{array}$ & $\begin{array}{c}\text { Number of overlapped } \\
\text { cascades }\end{array}$ & $\begin{array}{c}\text { Box size } \\
\left(\mathrm{a}_{0}{ }^{3}\right)\end{array}$ & $\begin{array}{c}\text { Number of } \\
\text { atoms }\end{array}$ \\
\hline $\begin{array}{c}\mathrm{Fe}-10 \\
\text { at.\% } \mathrm{Cr}\end{array}$ & 10 & 300 & 10 & $50 \times 50 \times 50$ & 250,000 \\
$\mathrm{Fe}-15$ & 10 & 600 & 10 & $50 \times 50 \times 50$ & 250,000 \\
at.\% $\mathrm{Cr}$ & 10 & 600 & 10 & $50 \times 50 \times 50$ & 250,000 \\
\hline
\end{tabular}




\section{Figure captions}

Figure 1. The defect distributions created by cascade overlap in the Fe-10 at.\% Cr system at 300 $\mathrm{K}$, where the green and blue spheres represent $\mathrm{Fe}$ and $\mathrm{Cr}$ interstitials, respectively, and the red ones represent vacancies.

Figure 2. The number of Frenkel pairs produced as a function of the number of the overlapped cascades in $\mathrm{Fe}-10$ at.\% $\mathrm{Cr}$ and $\mathrm{Fe}-15$ at.\% $\mathrm{Cr}$ systems at different temperatures.

Figure 3. The number of vacancy clusters and the corresponding number of vacancies in each vacancy cluster as a function of number of the overlapped cascades in $\mathrm{Fe}-10$ at.\% $\mathrm{Cr}$ and $\mathrm{Fe}-15$ at.\% Cr systems.

Figure 4. Ordered parameters as a function of number of the overlapped cascades in $\mathrm{Fe}-\mathrm{Cr}$ alloys: (a) first shell SRO parameter, (b) second shell SRO parameter, and (c) the coordination number-weighted average of the first and second SRO parameters.

Figure 5. The variation of ordering as functions of Metropolis Monte Carlo simulation timesteps in Fe-Cr alloys: (a) first shell SRO parameter, (b) second shell SRO parameter, and (c) the coordination number-weighted average of the first and second SRO parameters.

Figure 6. Distribution of $\mathrm{Cr}$ atoms near the largest vacancy clusters in $\mathrm{Fe}-\mathrm{Cr}$ alloys: (a) after cascade overlap in Fe-10 at.\% Cr system at 300K, (b) after $2 \times 10^{5} \mathrm{MMC}$ steps in $\mathrm{Fe}-10$ at.\% $\mathrm{Cr}$ system at $300 \mathrm{~K}$, (c) after cascade overlap in Fe-10 at. $\% \mathrm{Cr}$ at $600 \mathrm{~K}$, (d) after $2 \times 10^{5} \mathrm{MMC}$ steps in Fe-10 at.\% Cr at 600K, (e) after cascade overlap in Fe-15 at.\% Cr at $600 \mathrm{~K}$, and (f) after $2 \times 10^{5}$ MMC steps in $\mathrm{Fe}-15$ at.\% $\mathrm{Cr}$ at $600 \mathrm{~K}$. The blue and red spheres represent $\mathrm{Cr}$ atoms and vacancies, respectively. The yellow and green circles indicate the $\mathrm{Cr}$ precipitates formed near the vacancy clusters and Cr clusters. 


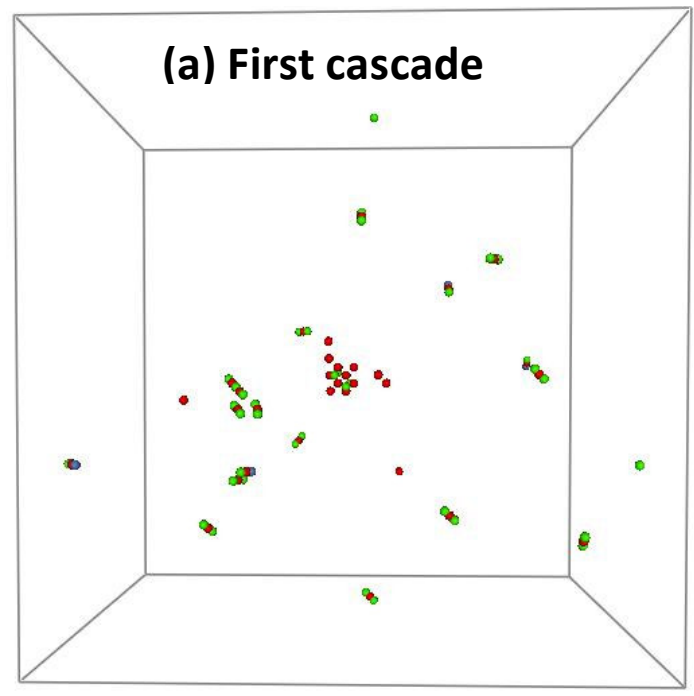

(b) Seventh cascade
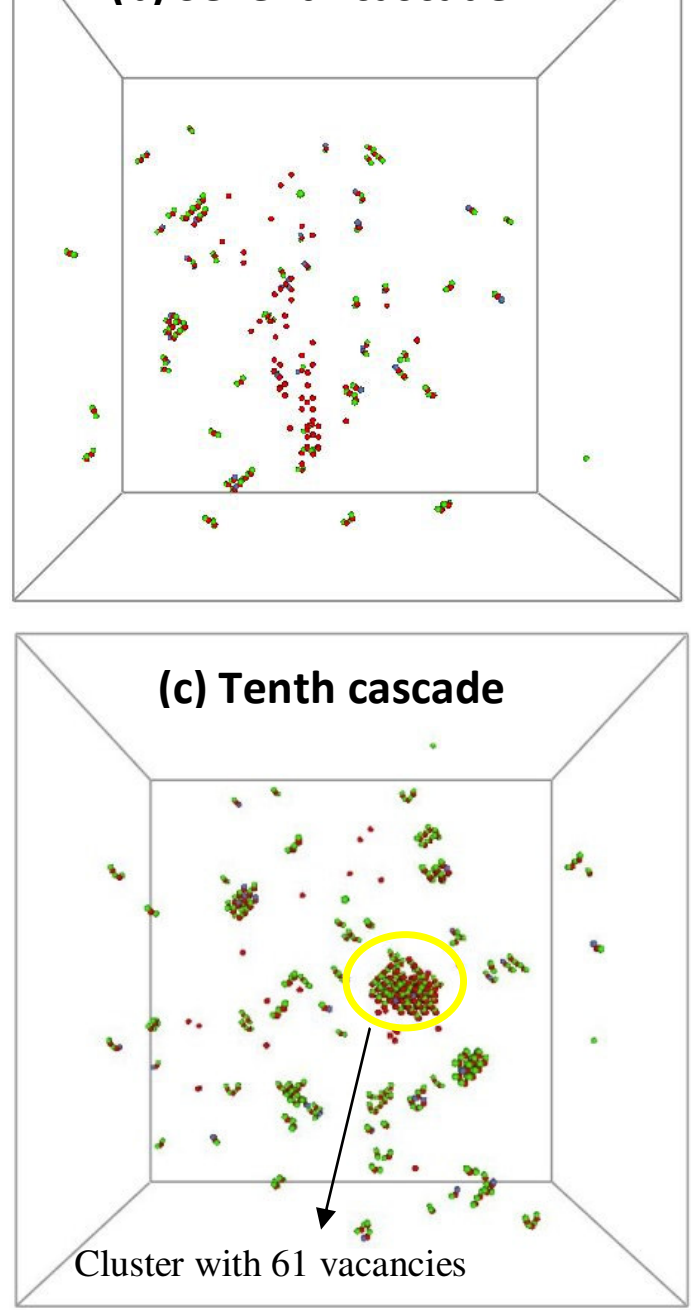

Figure 1 


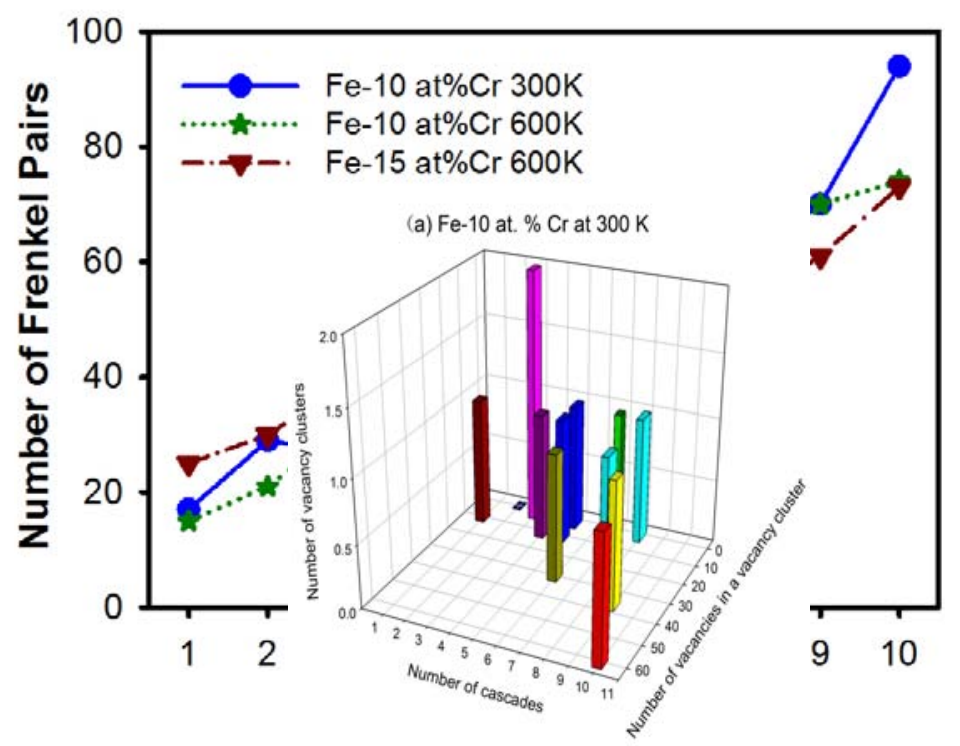

(b) $\mathrm{Fe}-10$ at. $\% \mathrm{Cr}$ at $600 \mathrm{~K}$

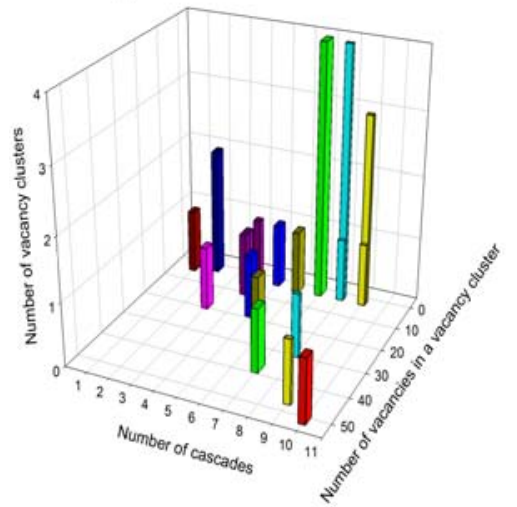

(c) $\mathrm{Fe}-15$ at. $\% \mathrm{Cr}$ at $600 \mathrm{~K}$

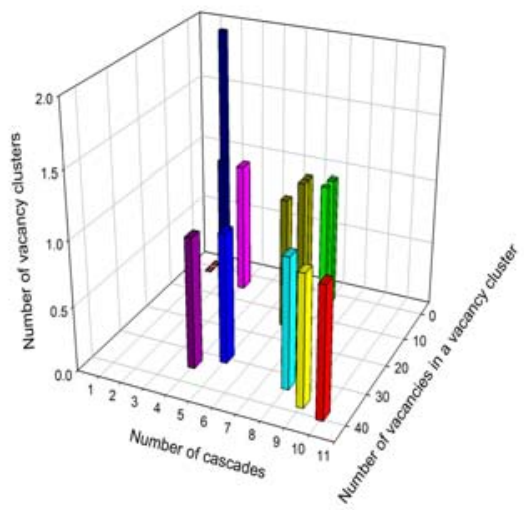


Figure 3
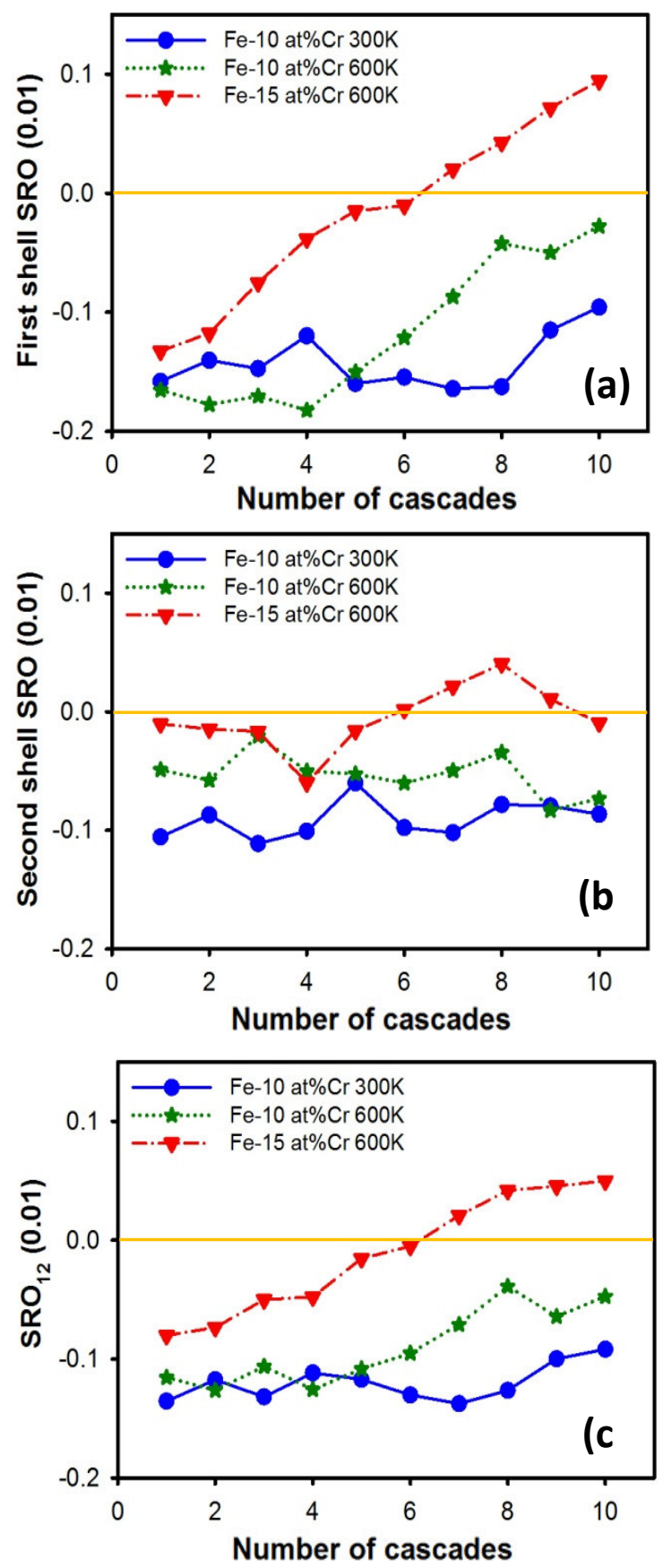

Figure 4 

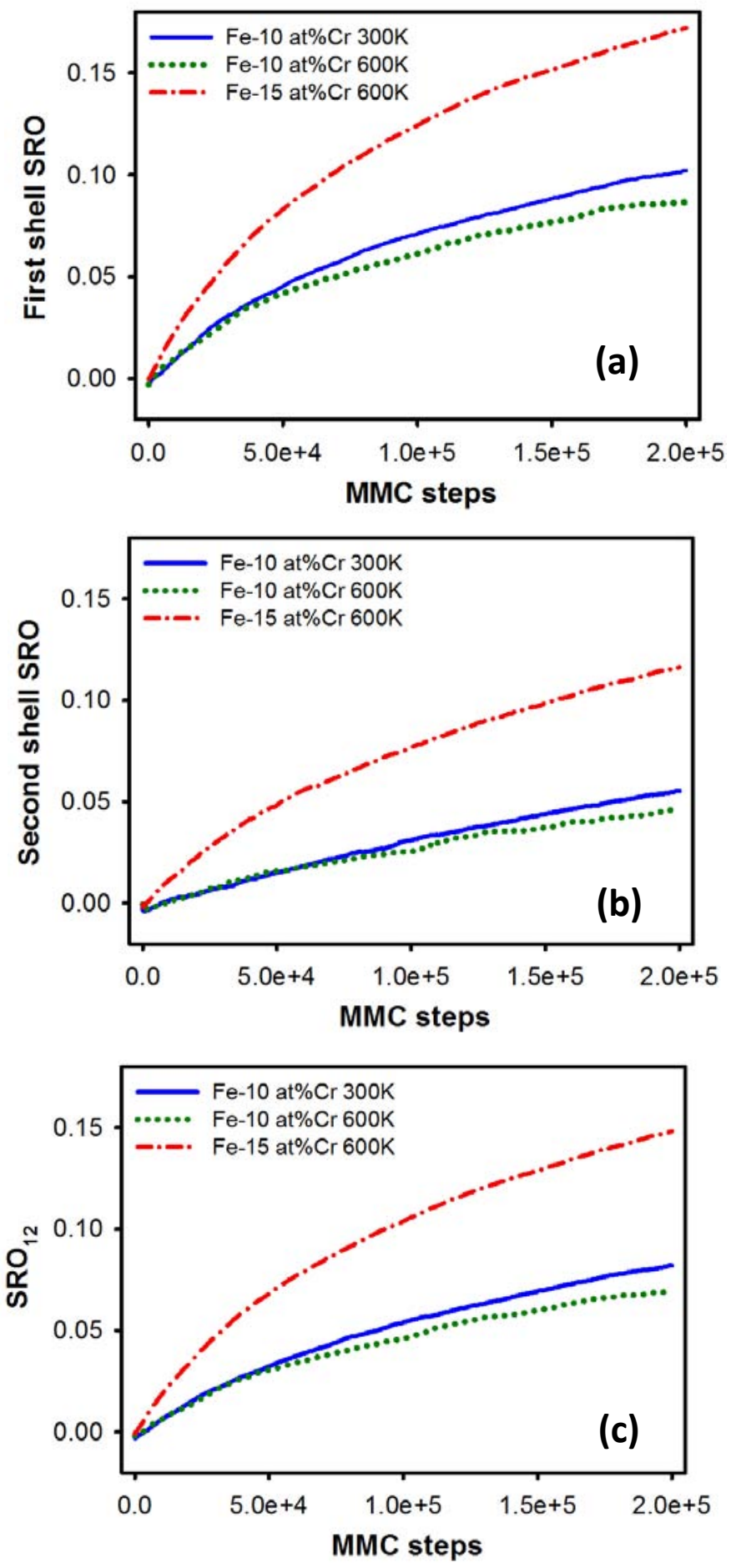

Figure 5 

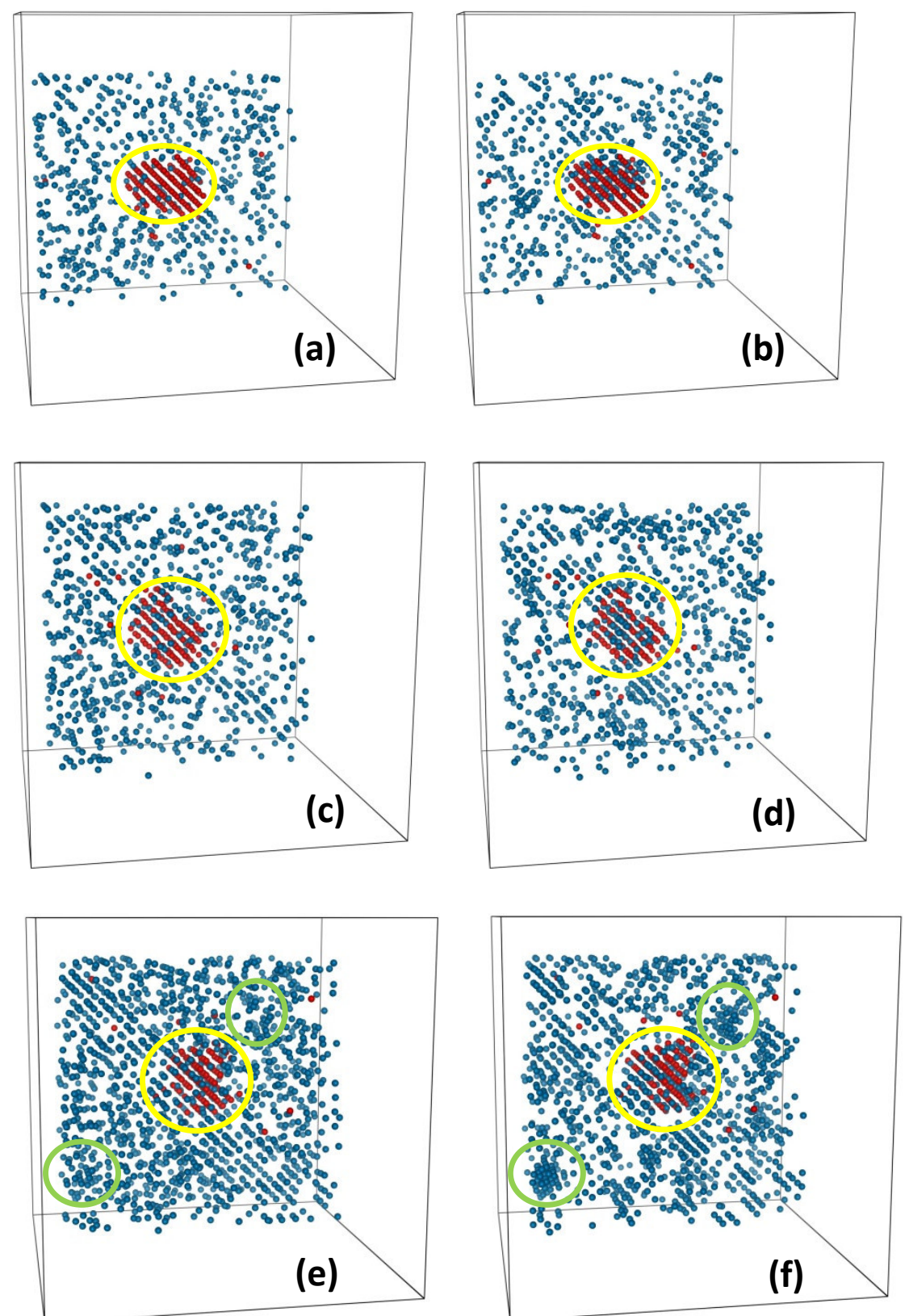

Figure 6. 
- $\mathrm{MD}$ and $\mathrm{MMC}$ methods are combined to study the nucleation of $\mathrm{Cr}$ precipitates.

- The number of Frenkel pairs increases with increasing the overlapped cascades.

- Cascade overlap could promote the formation of $\mathrm{Cr}$ precipitates in $\mathrm{Fe}-\mathrm{Cr}$ alloys.

- $\mathrm{Cr}$, vacancy clusters in overlapped cascades enhance nucleation of $\mathrm{Cr}$ precipitates. 


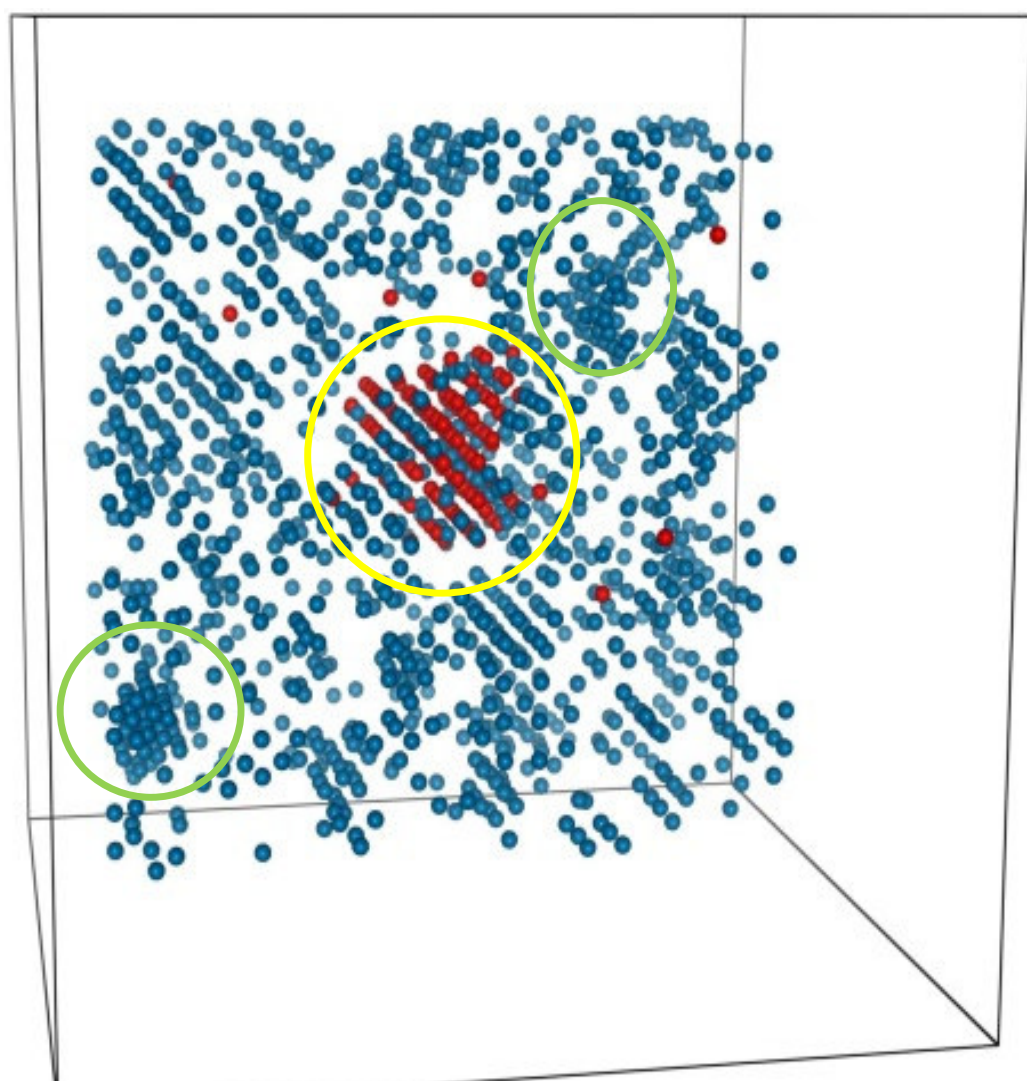

